\title{
OPEN Optical fibre-based single photon source using InAsP quantum dot nanowires and gradient-index lens collection
}

\author{
David B. Northeast ${ }^{1,3 凶}$, Dan Dalacu ${ }^{1,3}$, John F. Weber ${ }^{1}$, Jason Phoenix ${ }^{1,2}$, Jean Lapointe ${ }^{1}$, \\ Geof C. Aers ${ }^{1}$, Philip J. Poole ${ }^{1} \&$ Robin L. Williams ${ }^{1}$ \\ We present a compact, fibre-coupled single photon source using gradient-index (GRIN) lenses and \\ an InAsP semiconductor quantum dot embedded within an InP photonic nanowire waveguide. A \\ GRIN lens assembly is used to collect photons close to the tip of the nanowire, coupling the light \\ immediately into a single mode optical fibre. The system provides a stable, high brightness source of \\ fibre-coupled single photons. Using pulsed excitation, we demonstrate on-demand operation with \\ a single photon purity of $\mathbf{9 8 . 5 \%}$ when exciting at saturation in a device with a source-fibre collection \\ efficiency of $35 \%$ and an overall single photon collection efficiency of $10 \%$. We also demonstrate "plug \\ and play" operation using room temperature photoluminescence from the InP nanowire for room \\ temperature alignment.
}

Non-classical photon states (e.g. single, indistinguishable, or entangled) form the basis of photonic quantum technologies ${ }^{1}$. For implementation of these technologies in a future quantum network, the efficient transmission of these states between nodes in the network is critical. The most convenient transmission channel is an optical fibre, and in particular, a single-mode fibre (SMF) where photons exist in a single spatial mode. Solid-state twolevel systems (e.g. quantum dots, or defects) can produce the required states efficiently and deterministically, but the devices are typically designed to emit into free-space. High efficiency coupling of the free-space mode to the Gaussian mode of a SMF can be achieved with external optics ${ }^{2-5}$ and, using nearly perfectly matched modes, coupling efficiencies of $93 \%$ have been demonstrated ${ }^{3}$. To eliminate stability issues related to the alignment of free-space optics, new techniques for coupling to fibres are required. One can, for example, use the cleaved facet of a fibre to form one mirror of a Bragg reflector-based open microcavity allowing for direct collection of the cavity photons into the fibre ${ }^{6,7}$. Alternatively, evanescent coupling via tapered fibres can be applied to collect light from emitters ${ }^{8,9}$, cavities ${ }^{10,11}$ or tapered nanobeam waveguides ${ }^{12-14}$. Although near-unity coupling efficiencies are predicted, measured efficiencies remain substantially below those obtained using free-space optics.

The long term goal of fibre-based quantum light source development is a fixed-alignment device in a compact and robust package that can be operated in a turn-key manner. Many techniques have been proposed to eliminate user alignment by permanently attaching a fibre to the source; a so-called fibre-based plug and play $(\mathrm{PnP})$ source. One PnP approach involves positioning a fibre relative to an emitter using various alignment techniques, followed by gluing the fibre in place with epoxy. Initial experiments did not implement deterministic alignment, relying instead on a fibre bundle or array simply pressed to a sample containing emitters, e.g. planar dots ${ }^{15}$ or dots in weak cavity micropillars ${ }^{16,17}$ and measuring each fibre until one that is aligned to an emitter is found. An interferometric technique for aligning to the micropillar can be employed for deterministic coupling ${ }^{18}$ but ultimate efficiencies are only $18.6 \%$ due to the mode mismatch between the pillar and low numerical aperture (NA) SMF fibres. Slightly higher ultimate efficiencies are predicted using microlenses etched around pre-selected quantum dots ${ }^{19}$. In this case, the fibre is aligned using the host semiconductor photoluminescence from a gold aperture lithographically patterned around the microlens using alignment marks. To further increase the spatial overlap with the fibre mode, a miniaturized double-lens system is patterned around the microlens using a 3D, two-photon direct laser system, complete with a chuck for fibre placement ${ }^{20}$. Although relatively high coupling was achieved between the light emitted from the microlens and the fibre, the overall single photon collection

\footnotetext{
${ }^{1}$ National Research Council Canada, Ottawa, ON K1A 0R6, Canada. ${ }^{2}$ University of Waterloo, Waterloo N2L 3G1, Canada. ${ }^{3}$ These authors contributed equally: David B. Northeast and Dan Dalacu. ${ }^{\square}$ email: david.northeast@ nrc-cnrc.gc.ca
} 
efficiency was low $(0.56 \%)$ and attributed to a misalignment of the dot to the microlens. Finally, for devices employing high Q cavities ${ }^{21,22}$, active alignment between the fibre and cavity is possible at room temperature by measuring the transmission through or reflection from the cavity using two fibres, one on each side of the chip, which are subsequently glued in place. Measured cavity-fibre coupling efficiencies are high at $85 \%$ and close to the predicted values of $90 \%$. Measured single-photon collection efficiencies, however, were lower by a factor of 17 due to misalignment of the quantum dot within the cavity ${ }^{22}$.

A second approach relies on positioning an emitter on the facet of a cleaved fiber, where various techniques can be used to align to the fibre core ${ }^{23-26}$. This near-field coupling approach may have potential with defecttype sources e.g. nitrogen-vacancies in diamond nanocrystals ${ }^{23}$ or defects in boron nitride flakes ${ }^{24}$, but is not well suited to epitaxial quantum dots ${ }^{25,26}$ which are embedded in a host semiconductor. A more sophisticated approach to attaching a quantum dot-based source to a fibre facet relies on nanowire structures ${ }^{27}$ where the nanowire geometry is used to improve mode-matching. Alternatively, instead of a cleaved facet, tapered fibre tips can used ${ }^{28}$. In this case, the emitter is located within a tapered nanobeam waveguide which is removed from the growth substrate and placed on the fibre tip, where it couples to the fibre evanescently, in a similar manner to the tapered fibre approaches described above.

In Table 1 we summarize the performance of sources that have been operated in a PnP mode. From the table, it is clear that several approaches can provide good overlap between the optical modes of the source and the fibre, with predicted coupling efficiencies approaching $100 \%$. In practice, however, devices dramatically under-perform. Calculated efficiencies typically assume perfect alignment of the fibre and source and ideally this would be done actively using emission from the emitter. Many emitters, however, are insufficiently bright at room temperature (where epoxies are applied) to allow for active alignment to the emitter emission. Instead, alignment is performed either using apertures containing the emitter ${ }^{19}$ or using photonic structures within which the ideal positioning of the emitter is not guaranteed ${ }^{18,20,22}$.

We report here on a fibre-coupled, single photon source based on a quantum dot in a bottom-up grown photonic nanowire waveguide and a compact, double gradient-index lens (GRIN) system. The bottom-up approach guarantees optimal positioning of the quantum dot within the photonic nanowire waveguide ${ }^{29}$, with demonstrated coupling of the dot emission to the fundamental $\mathrm{HE}_{11}$ mode of $\beta=0.86^{30}$, close to the ultimate value of $\beta \sim 0.95^{31}$. By appropriately tapering the photonic nanowire, a Gaussian mode is transformed to free-space for perfect mode-matching to a SMF fibre ${ }^{3}$. We first demonstrate efficient coupling between the nanowire source and the GRIN lens system at $4 \mathrm{~K}$ in a stable, closed-cycle cryostat where the dot emission is used for alignment. We then demonstrate PnP operation by permanently fixing the GRIN lens system to the nanowire sample. In this case, we use photoluminescence from the band to band recombination in the photonic nanowire surrounding the quantum dot for aligning. Unlike the dot, emission from the host nanowire remains bright at room temperature and, importantly, the dot and nanowire are spatially co-located allowing for indirect room temperature $X-Y$ alignment of the source and the GRIN lens system.

\section{Optical measurements}

The single photon sources used in this work are InAsP quantum dots that are embedded within InP nanowires. After processing, nanowires have a typical radius of $\sim 250 \mathrm{~nm}$ and are $\sim 10$ microns long, see Fig. 1c. The growth method allows for the definition of a tapered tip with controlled angle, suitable for launching photons in a Gaussian mode ${ }^{3}$ into free space, as mentioned above.

Light collection from the nanowire utilizes two GRIN lenses and a pig-tailed fibre. Emission from the nanowire is collimated with a $0.5 \mathrm{NA}$ GRIN lens and re-focused into the fibre using a second lens with NA $=0.2$. The schematic of the GRIN assembly is shown in Fig. 1b. The system is designed for an NA that matches the divergence of the $\mathrm{HE}_{11}$ mode emitted from the tapered photonic nanowire waveguide ${ }^{32}$ in order to provide a high mode overlap and near unity collection, $\eta_{\text {calculated }} \sim 100 \%$. Low temperature photoluminescence spectra of the quantum dot sample were taken with the GRIN lenses in a closed-cycle He cryostat using the system arrangement seen in Fig. 1a.

In lieu of conventional imaging, which is not possible using the GRIN lens assembly, we perform two dimensional (2D) scans of the sample surface using the $X-Y$ piezoelectric stage controllers. The scans are performed using a two stage process. We first find the focus $(Z)$ of the GRIN lens assembly by maximizing the reflected power from a $930 \mathrm{~nm}$ laser (similar to the dot emission wavelength). At this $Z$ position the GRIN lens assembly is focused on the substrate which is sufficient to observe emission from both the quantum dot and the InP making up the photonic nanowire. The latter adopts a wurtzite crystal phase in our nanowires, with a higher bandgap (832 $\mathrm{nm}$ at $4 \mathrm{~K}$ ) compared to bulk $\mathrm{InP}$ which has a zincblende crystal structure. In the second stage, we excite above band with wavelengths of $780 \mathrm{~nm}, 632 \mathrm{~nm}$, or $670 \mathrm{~nm}$ and record the photoluminescence as a function of $X-Y$ position. A typical spectrum obtained when the GRIN lens assembly is over a nanowire is shown in Fig. 1e, with both InAsP dot emission and wurtzite InP emission clearly visible. Fig. $1 \mathrm{~d}$ is a plot of count rates as a function of laser power (normalized to the power at saturation, $P_{\text {sat }}$ ).

Initial scans locate the nanowires on the substrate and we use these to adjust the $Z$-focus to optimize the collection of the quantum dot emission as opposed to focusing on the substrate. Examples of such in-focus scans can be seen in Fig. 2. The position step size used in the scans is $\sim 0.5 \mu \mathrm{m}$ in $X$ and $Y$, and the mode of travel of the piezoelectric drives is with slip-stick motion. Each of these coloured pixels is derived from a full spectrum measurement shown in Fig. 1d. In Fig. 2a, we take the maximum count rate in the expected spectral range of the quantum dot emission (908-950 nm) and in Fig. 2 b we take a sum of the counts in the spectral range $823-836 \mathrm{~nm}$ which contains the peak associated with band to band recombination in the wurtzite InP nanowire. Comparison of the two figures highlights the spatial co-location of the dot and nanowire emission, important for room temperature alignment discussed later. 
(a)

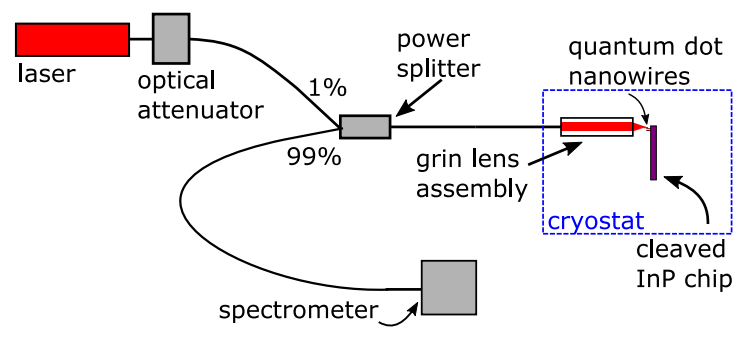

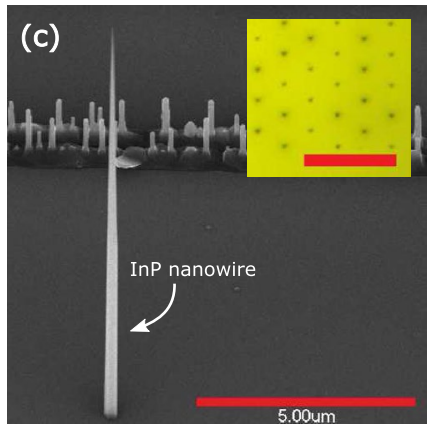

(d)

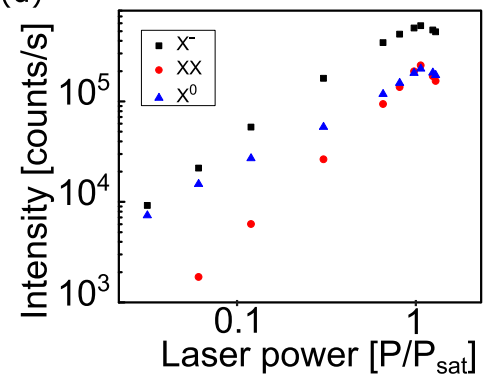

(b)
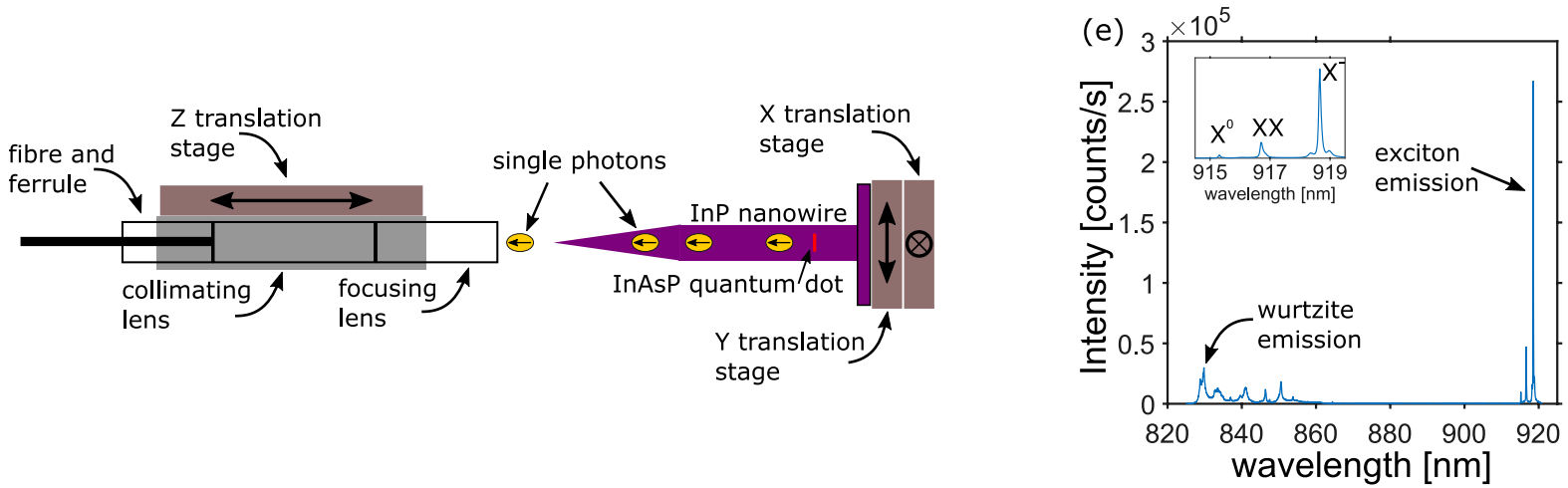

Figure 1. The optical system for detecting spectral response is shown in (a). A closer view of the GRIN lens arrangement is seen in $(\mathbf{b})$. The nanowire sample can be translated in two dimensions $(X$ and $Y$ ), and the GRIN lens may be moved to change the focus of the lens relative to the nanowire $(Z)$. The grey box overlapping the GRIN lenses and fibre ferrule is a ferrule sleeve used to maintain optical alignment. An SEM image of a nanowire can be seen in (c). Inset is a top-down optical image of the hexagonal grid of nanowires-red bar represents $20 \mu \mathrm{m}$-used in subsequent scan images. An intensity saturation curve of the emission from the quantum dot, (d), shows the laser pump dependence. The spectral plot in (e) shows a bright $\mathrm{X}^{-}$exciton emission line in a typical quantum dot used in this work. Inset in (e) is a plot capturing only the quantum dot emission (with labelled complexes).

\begin{tabular}{|l|l|l|l|l|}
\hline Material system & Alignment & $\eta_{\text {calculated }}$ & $\eta_{\text {measured }}$ & Ref. \\
\hline QD- $\mu$ pillar & Fibre bundle & $0.69 \%$ & $0.095 \%$ & 15 \\
\hline QD- $\mu$ pillar & Fibre array & $26 \%$ & - & 17 \\
\hline QD- $\mu$ pillar & Reflection & $18.6 \%$ & - & 18 \\
\hline QD-nanowire & Visual & $9.2 \%$ & $5.8 \%$ & 27 \\
\hline QD-nanowire & QD PL & - & - & 26 \\
\hline QD-flake & Random & - & - & 25 \\
\hline BN-flake & Visual & $10.15 \%$ & - & 24 \\
\hline NV-nanocrystal & AFM & $100 \%$ & - & 23 \\
\hline QD-waveguide & Visual & $88 \%$ & $1.4 \%$ & 28 \\
\hline QD- $\mu$ lens & AM \& bulk PL & $18 \%$ & $0.28 \%$ & 19 \\
\hline QD- $\mu$ lens & AM \& Fibre chuck & $81.8 \%$ & $0.56 \%$ & 20 \\
\hline QD-cavity & Transmission & $90 \%$ & $5 \%$ & 22 \\
\hline
\end{tabular}

Table 1. Quantum light sources operated in PnP mode. QD quantum dot, $N V$ nitrogen vacancy, $B N$ boron nitride, $P L$ photoluminescence, $A M$ alignment marks, $A F M$ atomic force microscope, $\eta_{\text {calculated }}$ calculated efficiency assuming unity outcoupling from emitter, $\eta_{\text {measured }}$ measured single photon collection efficiency into fibre.

To determine the purity of the gathered single photons, measurements of the second order correlation function, $g^{(2)}(\tau)$, were taken in a typical Hanbury Brown and Twiss (HBT) arrangement. We first optimize the position of the GRIN lens using the $X-Y-Z$ stages for maximum collection of emission from a chosen quantum dot. Count rates at saturation using continuous wave $(\mathrm{CW})$ excitation are up to 1.4 Mcps. Figure 3a shows a CW $g^{(2)}(\tau)$ measurement made using a HeNe laser as an above-band excitation source. Assuming the CW $g^{(2)}(\tau)$ 

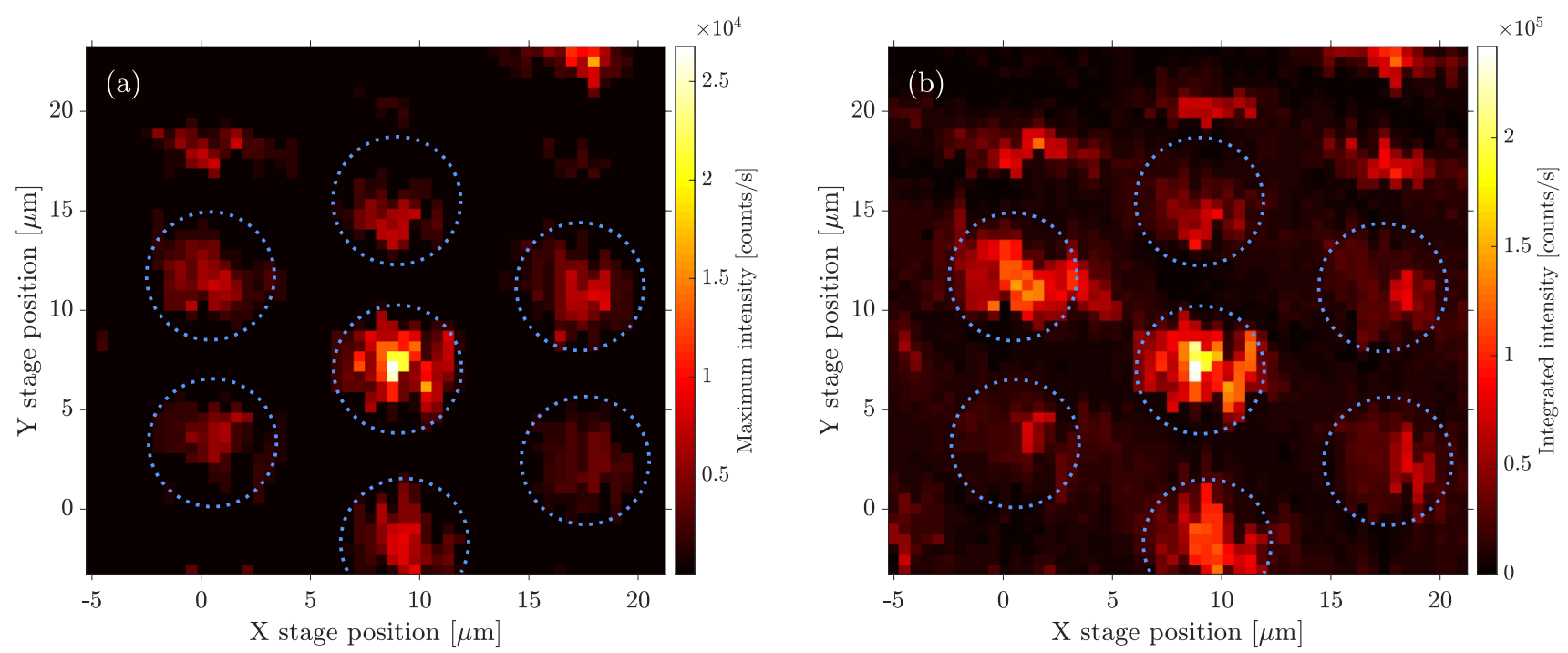

Figure 2. Plots of quantum dot and wurtzite InP emission as a function of GRIN lens position relative to the sample substrate. (a, b) show data from a $25 \times 25 \mu \mathrm{m}^{2}$ scan of a hexagonal array of nanowires (see inset image in 1c), with a scan step size of $0.5 \mu \mathrm{m}$. The quantum dot count rate represents the maximum value in a spectral range of $908-950 \mathrm{~nm}$, which is the expected range for this sample. The wurtzite InP emission in (b) is an integrated count from 823 to $836 \mathrm{~nm}$. Blue dotted circles are used to emphasize the same subsection of the hexagonal array of nanowires.

without the effect of detectors is given by a Laplace distribution and that the detector response can be described by a Gaussian, the normalized second order correlation measurement seen in the experiment is given by

$$
g^{(2)(\tau)}=1-\frac{A}{2}\left(B_{+}+B_{-}\right)
$$

where $B_{ \pm}$is defined as

$$
B_{ \pm}=\left[1 \mp \operatorname{erf}\left(\frac{2 \tau \pm \xi^{2} \Gamma}{2 \xi}\right)\right] e^{ \pm 4 \tau / \Gamma+\xi^{2}}
$$

where $\Gamma$ is the radiative recombination rate of the excitonic complex, $\xi$ is the detector jitter, and $A$ describes the magnitude of the correlation minimum. The SPADs used in these measurements have a detection jitter of $\xi \approx 190 \mathrm{ps}$. The expected time-correlated response without the influence of detectors is also plotted against the experimental data. This curve is produced by taking the fit to the correlation data and plotting it again with $\xi=0$.

In Figure $3 \mathrm{~b}$ we demonstrate on-demand operation using a diode laser (emitting at $670 \mathrm{~nm}$ ) as a pulsed excitation source. Pumping at saturation using 39 ps pulses repeated at $40 \mathrm{MHz}$, we obtain a total count rate at the detectors of 0.2 Mcps. At this pump power, the measured $g_{\text {sat }}^{(2)}(0)=0.015$ (i.e. the normalized area of the $\tau=0$ peak). The same value was obtained by fitting the $g^{(2)}(\tau)$ curve using a stochastic model to simulate the pulsed HBT experiment ${ }^{33}$. The high single photon purity, even at saturation and using above-band excitation, is a consequence of the deterministic growth process employed in the manufacture of the source, which produces devices containing one and only one emitter.

Single photon indistinguishability measurements, using our InAsP quantum dots in nanowires, have been previously reported ${ }^{30}$. These used a free-space setup to measure Hong-Ou-Mandel visibilities as high as $85 \%$, with a $100 \mathrm{mK}$ environment. Here we focus on photon collection, and note that improvements to indistinguishability will likely require an arrangement including cavity enhancement.

To estimate the device efficiency, $\eta_{\text {device}}$, we account for the throughput of all the fibre components $(20 \%)$ and the detector efficiency (25\%) from which we calculate 4 Mcps single photons coupled into the fibre by the GRIN lens. Considering the excitation pulse repetition rate of $40 \mathrm{MHz}$, and assuming the quantum efficiency for the quantum dot is $100 \%$, this corresponds to $10 \%$ of the photons emitted by the dot being coupled into the fibre. To estimate the collection efficiency of the GRIN lens system itself we have to take into account the fact that not all the photons emitted by the dot will be radiated from the top of the nanowire. We assume a loss of $50 \%$ for photons emitted by the dot that are directed down towards the substrate and $5 \%$ into radiation modes (i.e. $\beta=95 \%$ ). We also have to take into account $25 \%$ which emit from a different charge state (see Fig. 1c) and $20 \%$ that emit into the phonon sidebands, both of which are filtered out. These loss channels reduce the maximum number of the desired photons at the input of the GRIN lens to $28.5 \%$ of $40 \mathrm{MHz}$. Accordingly, we estimate a GRIN lens assembly collection efficiency of $35 \%$ (i.e. $\eta_{\text {measured }}$ in Table 1 ).

We speculate that the large fraction of uncollected photons (65\%) is due to imperfect alignment, in particular, a tilt of the GRIN lens system with respect to the substrate. In a separate experiment, we have measured the dependence of the coupling efficiency on the deviation of the GRIN lens axis from the substrate normal 
(a)

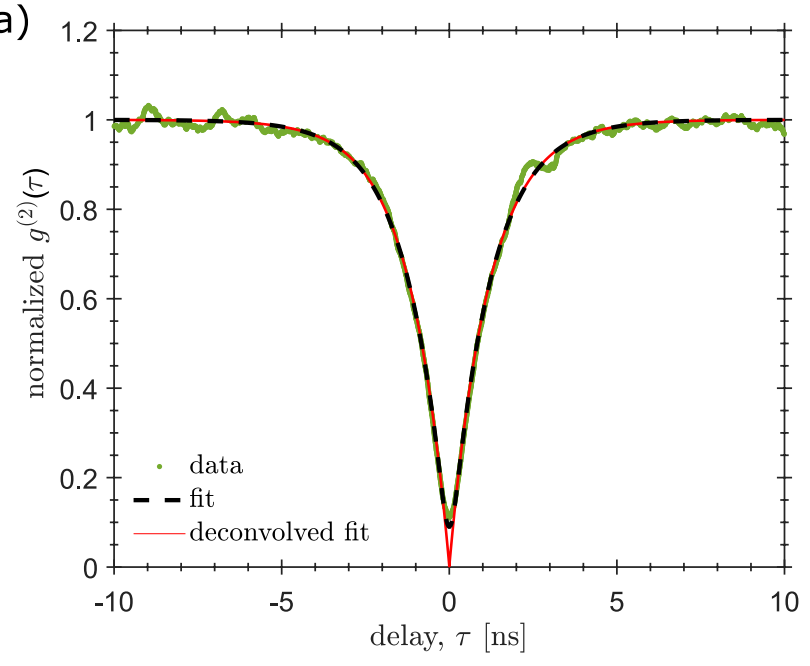

(c)

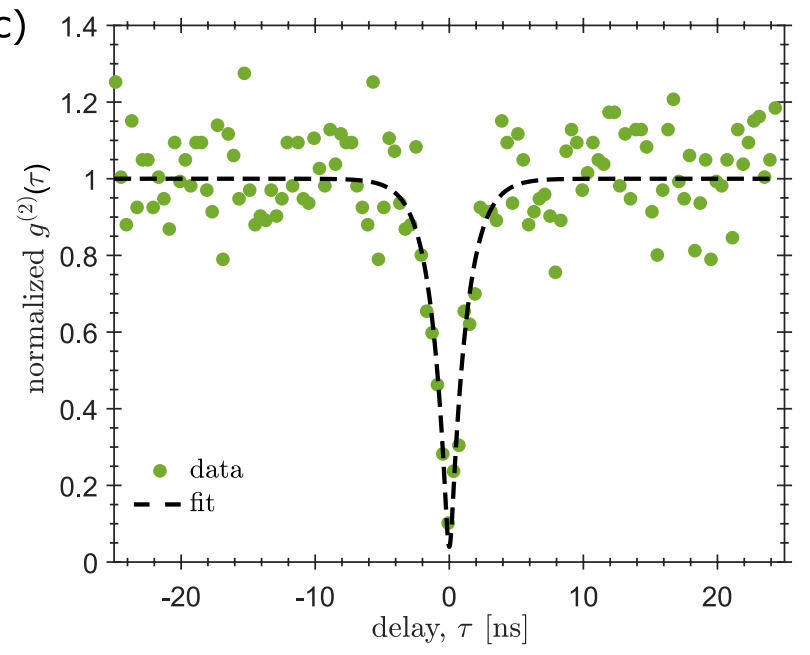

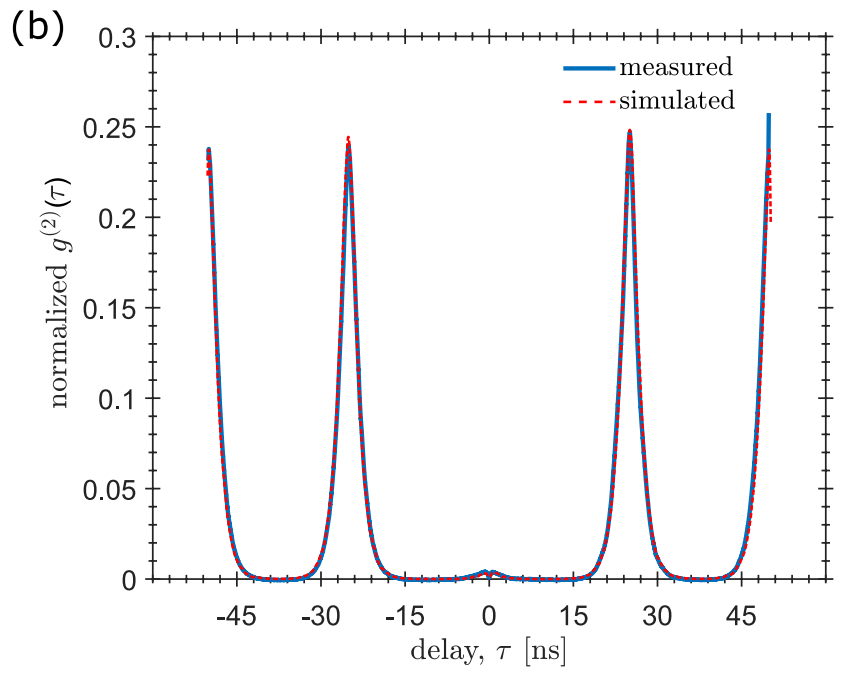

(d)

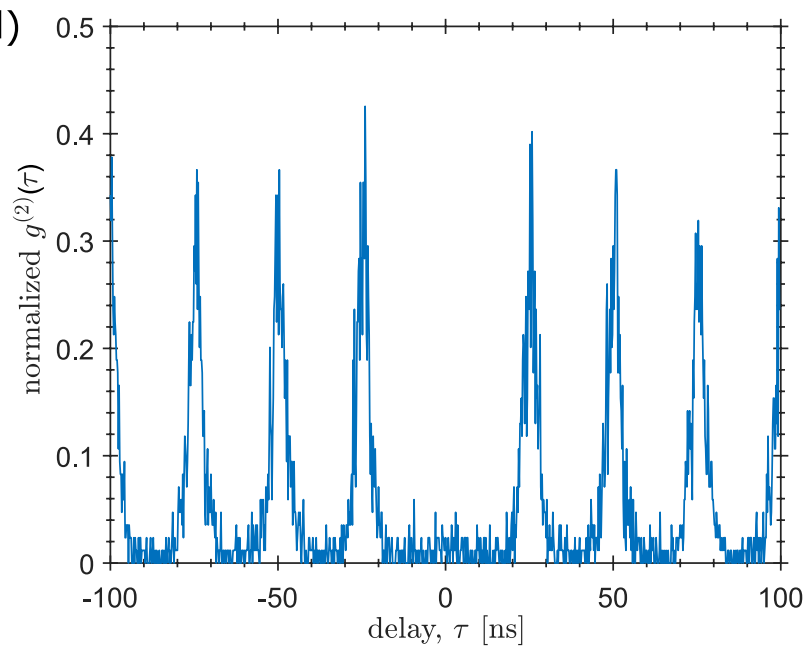

Figure 3. $g^{(2)}(\tau)$ measurements made with active $(\mathbf{a}, \mathbf{b})$ and fixed $(\mathbf{c}, \mathbf{d})$ alignment. In $(\mathbf{a}, \mathbf{c})$, a CW laser (HeNe, $633 \mathrm{~nm}$ ) is used to excite the quantum dot at saturation, with $g^{(2)}(0)=0.002$ and 0.04 , respectively, after deconvolving the detector response jitter using Eq. (1). In (b, d), the measurements were made using pulsed excitation with a pulsed diode laser (PDL) at $670 \mathrm{~nm}$. A pulsed $g^{(2)}(\tau)=0.015$ is estimated in (b) through comparison to simulated data which is also plotted. The two sets of data (active and fixed alignment) were measured on different nanowire quantum dot devices.

and have observed a decrease of over $50 \%$ at angles as small as $1^{\circ}$. Angle alignment can be readily improved by incorporating precise pitch and yaw control during the alignment process. This change would entail adding two additional piezoelectric stages to the system, moving either the lenses or InP substrate.

We also note that nanowire device designs exist that can reduce some of non-alignment related sources of loss, including the incorporation of a back mirror ${ }^{31,34}$ and charge-state control ${ }^{35}$. The latter reference describes a scenario where four metallic gates, deposited near to a nanowire, could allow quadrupole fields to manipulate the quantum dot and its properties (such as the fine structure splitting of the neutral exciton). Additionally, resonant pumping schemes are compatible with the same GRIN lenses that collect the single photon emission. Coherent excitation, together with charge-state control, can reduce the losses associated with above-band pumping exciting unwanted charge states.

\section{Fixing alignment}

We turn next to room temperature alignment and PnP operation. We focus a $930 \mathrm{~nm}$ laser onto the surface looking at the reflected laser power, as before, but here, we also perform an $X-Y$ scan which provides information on the surface topography. An example is shown in Fig. $4 \mathrm{~b}$ where the target array of nanowires is clearly visible. While knowing the location of nanowires using reflected laser power can give a rough stage location for GRIN lens-nanowire coupling, it is not suitable for determining if the lens is in the proper focus position to maximize quantum dot emission collection. To better position the fibre and lenses, we investigated whether room temperature wurtzite InP emission scans were possible. In Fig. 4c, such a scan shows that wurtzite InP emission can clearly show distinct nanowires, allowing for precise positioning of the collection lenses at room temperature. An optical image of the same nanowire array (Fig. 4a) shows that the emission image accurately recreates the 

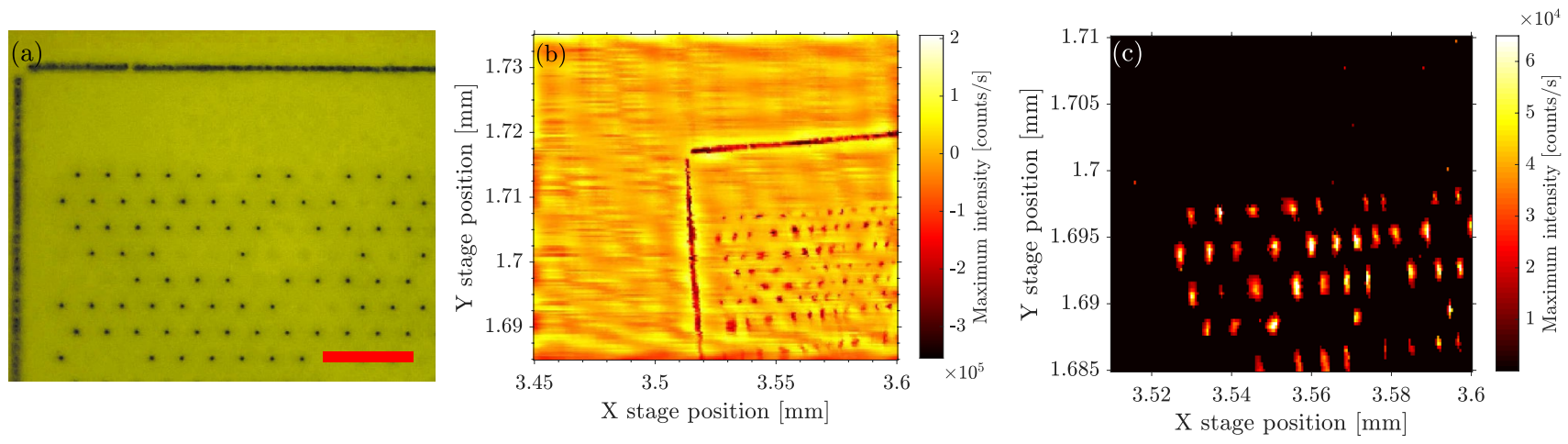

Figure 4. An optical image of a hexagonal array of nanowires, shown in (a), is compared with (b) a reflection $(930 \mathrm{~nm})$ and $(\mathbf{c})$ a wurtzite InP emission scan. The scans were performed at room temperature and atmospheric pressure. Nanowires are clearly seen and distinct in position, allowing the lens assembly to be accurately placed over any scanned nanowire. The red scale bar in (a) represents $20 \mu \mathrm{m}$.

(a)

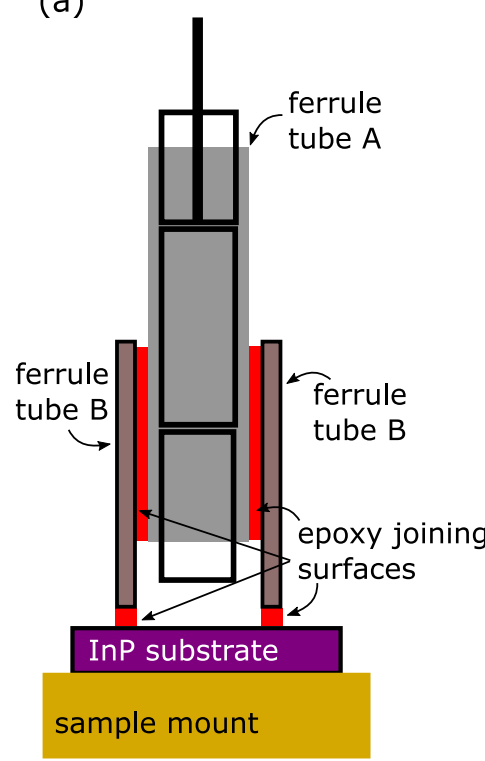

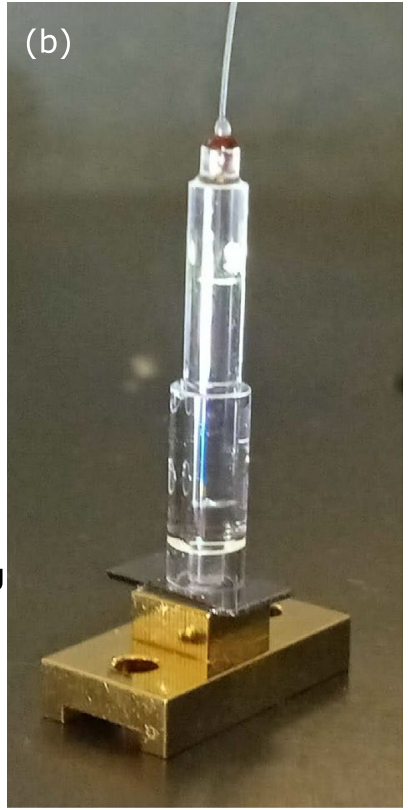

(c)

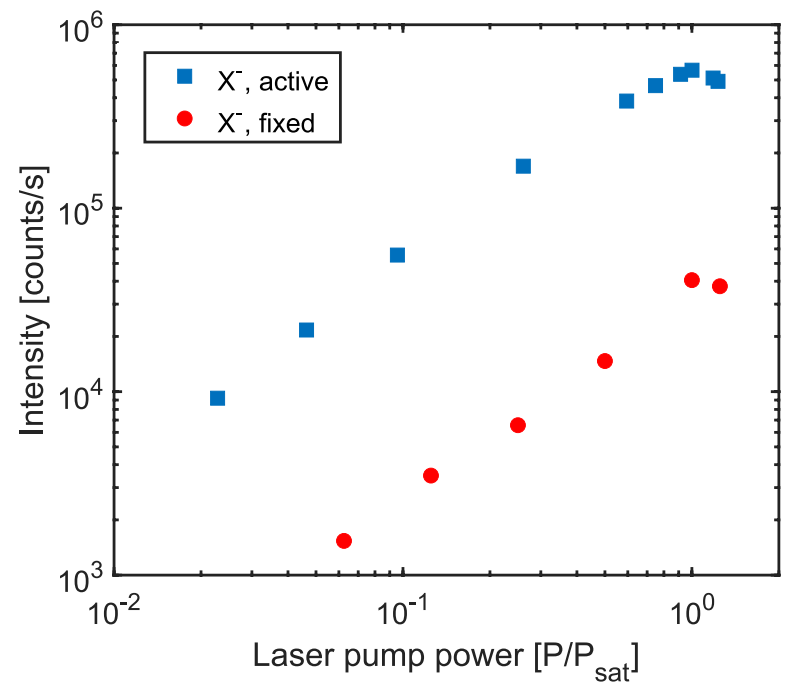

Figure 5. The fixed alignment structure is shown in a schematic representation, (a), and in a picture of a finished device, (b). The schematic highlights the critical surfaces that are bonded together with epoxy, after alignment to a nanowire is established. In (c), the change to the saturation count rate to the $X^{-}$complex-before and after fixing the alignment-shows an order of magnitude decrease.

same arrangement of nanowires, where missing wires are seen in the same locations in both images. The GRIN lenses and sample are fixed in place above a chosen nanowire. A picture of the finished device is shown in Fig. $5 \mathrm{~b}$.

The device was then cooled to $4 \mathrm{~K}$ and operated as a PnP source. Measured count rates were $\sim 10^{4} \mathrm{cps}$, significantly lower compared to devices employing active alignment at low temperature, which can be seen in contrasting saturation curves in Fig. $5 c$. The new count rate corresponds to an efficiency of $\eta_{\text {measured }}=0.7 \%$. Single photon purity remained high, with $g^{(2)}(0) \sim 0.01$, see Fig. $3 \mathrm{c}$ and d. This initial device was intended to verify the feasibility of the approach, and, in particular, the alignment stability. No variation in count rate was observed after several cool-downs in the cryostat, nor was any observed after several immersions in liquid $\mathrm{N}_{2}$.

We attribute the decrease in counts upon fixing the lens system to the substrate to a misalignment in the focus that occurs with room temperature InP emission alignment. In a separate experiment we found that the focus position for optimal collection of the InP emission occurs a few microns below that of the quantum dot emission. Below we identify several routes to increasing the count rate to the levels achieved using active low temperature alignment.

One approach requires more careful attention to the chosen focus position at which the GRIN lens is fixed. For example, the difference between the room temperature InP focus and low temperature dot focus can be accurately determined by fabricating a series of assemblies each fixed at a different focus. A more efficient approach entails using a multidot nanowire ${ }^{36}$ where each energy-tuned dot is incorporated at a different position along the the nanowire length. Once known, the focus offset can be compensated for when gluing the GRIN lens. Another 
interesting avenue is to apply the technique to telecom nanowire quantum dot emitters ${ }^{37}$. The quantum dots used in these sources have a deeper confining potential and emit brightly even at room temperature ${ }^{38}$. Consequently, emission from the dots themselves can be used for alignment instead of the InP nanowire emission.

Additionally, using modified nanowire geometries, NA values approaching 0.3 are predicted ${ }^{32}$. Devices based on such low NA devices offer a 3-fold relaxation in the tolerance of the position of the focus compared to $\mathrm{NA}=0.5$ devices. Applying the above modifications to the device design and alignment procedure together with the addition of pitch and yaw control during alignment can potentially lead to the realisation of PnP single photon sources with collection efficiencies approaching $\eta_{\text {calculated }}=100 \%$. Considering the recent demonstration of the high optical quality of the nanowire quantum dot system ${ }^{39}$, this approach may also be useful for producing indistinguishable photons provided that coherent pumping schemes are employed for excitation.

\section{Conclusion}

In summary, we demonstrate a process to efficiently couple single photons from InAsP quantum dots embedded in InP photonic nanowire waveguides into a single mode fibre using a compact and robust GRIN lens assembly. The collection efficiency of the GRIN lens system (e.g. fraction of the single photons directed towards the lens assembly that were collected) was $35 \%$ whilst the overall single photon collection efficiency (fraction of the total single photons produced by the quantum dot that were collected) was $10 \%$. We also describe a manufacturing method to produce high purity single photon sources that can be operated in a "plug and play" mode. The method is based on active alignment using the emission of the InP nanowire in which the InAsP quantum dot is embedded. This alignment can be performed at room temperature, where the fibre can be permanently attached to the chip.

\section{Methods}

Nanowire and quantum dot growth. We use site-selective vapour-liquid-solid (VLS) epitaxy; a process described in more detail in previous studies ${ }^{40,41} .20 \mathrm{~nm}$ gold particles are lithographically defined and deposited to provide a catalyst at desired growth sites. Along with $250 \mathrm{~nm}$ holes in $\mathrm{SiO}_{2}$ growth mask, the gold defines the InP nanowire diameter during the VLS epitaxy process. Nanowires are typically grown to a length of $\sim 750 \mathrm{~nm}$, and quantum dots are defined $\sim 500 \mathrm{~nm}$ above the substrate. At this point, a change to the growth conditions promotes substrate growth over catalyzed growth, and the nanowire radius increases to that defined by the holes in the oxide mask. The InP nanowire growth can be tailored to finish with wire lengths from $\sim 1 \mu \mathrm{m}$ to $50+\mu \mathrm{m}$.

GRIN lens assembly and GRIN lens-sample assembly. For the GRIN lens assembly (Grintech $\mathrm{GmbH}$ ), we estimate, at $950 \mathrm{~nm}$ wavelength, $\sim 220 \mu \mathrm{m}$ for the free space focal length, and $0.76 \mu \mathrm{m}$ for the beam waist. This is predicted using one dimensional calculations with the transfer matrix method. Using a $965 \mathrm{~nm}$ laser, a CCD camera is used to directly measure the beam waist as a function of camera-lens separation in the far field. Using $w(z) \approx \lambda\left(z-z_{0}\right) /\left(\pi n_{0} w_{0}\right)$, the waist was found to be $w_{0}=0.80 \mu \mathrm{m}$, corresponding to a numerical aperture of NA $=0.40$. The lens assembly is fixed together, and to the fibre (Nufern HI 1060), using an optically clear epoxy (EPO-TEK 301) and a glass ferrule sleeve.

To permanently fix the alignment of the nanowire to the fibre assembly we first fix the nanowire growth substrate onto the metal sample holder using 3M 2216 B/A epoxy adhesive. An outer ferrule tube (B in Fig. 5a) is placed, loose, on top of the InP substrate, located roughly above a target array of nanowires. The GRIN lens assembly used in the low temperature measurements (A in Fig. 5a) is then lowered into the outer ferrule tube. Using a $930 \mathrm{~nm}$ laser, the GRIN lens assembly is brought into focus on the substrate surface using the reflected intensity to optimize - this focus position is typically a few microns below the ideal focus position for quantum dot emission. The GRIN lens assembly (A) is now glued to the inner walls of the outer ferrule tube (B) using EPO-TEK 301-2 epoxy and cured for approximately $3 \mathrm{~h}$ at $75^{\circ} \mathrm{C}$. After curing, the assembly is raised a few microns and a small $X-Y$ scan is performed to locate a chosen nanowire using the wurtzite InP emission. Once located, the optimum focus is found and the outer ferrule tube is glued to the substrate using EPO-TEK 301-2 epoxy and further cured at $75^{\circ} \mathrm{C}$ for another $3 \mathrm{~h}$.

Photoluminescence spectroscopy and correlation measurements. The cryostat is equipped with three orthogonal piezoelectric-driven translation stages. The GRIN lens system is mounted on the $Z$ stage whilst the sample is on the $X-Y$ stages. The system is optically pumped using a fibre-coupled laser source connected to the $1 \%$ arm of a 99:1 fused fibre power splitter and focused on the sample by the GRIN lenses. Reflected laser light and sample emission are collected by the lens assembly and directed along the $99 \%$ arm of the power splitter, towards a spectrometer equipped with a CCD detector array.

To measure single photon purity, the emission from the quantum dot is first filtered by a tunable narrowband transmission filter. The filter has a $0.1 \mathrm{~nm}$ bandwidth, and suppresses $>45 \mathrm{~dB}$ of the out-of-band transmitted signal, including the laser pump power and unwanted sample emission. The filtered signal is fed into a 50:50 fused fibre power splitter with each $50 \%$ arm of the splitter leading to a single-photon avalanche diode (SPAD) detector. The signals from the detectors are time-correlated using counting electronics to build a histogram of the delay times between detection events on the first and second detector, $\tau=t_{2}-t_{1}$.

\section{Data availability}

The datasets generated during and/or analysed during the current study are available from the corresponding author on reasonable request. 
Received: 7 September 2021; Accepted: 11 November 2021

Published online: 24 November 2021

\section{References}

1. O’Brien, J. L., Furusawa, A. \& Vučković, J. Photonic quantum technologies. Nat. Photon. 3, 687 (2009).

2. Gazzano, O. et al. Entangling quantum-logic gate operated with an ultrabright semiconductor single-photon source. Phys. Rev. Lett. 110, 250501 (2013).

3. Bulgarini, G. et al. Nanowire waveguides launching single photons in a gaussian mode for ideal fiber coupling. Nano Lett. 7, 4102-4106 (2014).

4. Lee, C.-M. et al. Bright telecom-wavelength single photons based on a tapered nanobeam. Nano Lett. 21, 323 (2021).

5. Tomm, N. et al. A bright and fast source of coherent single photons. Nat. Nano. 16, 399 (2021).

6. Muller, A., Flagg, E. B., Metcalfe, M., Lawall, J. \& Solomon, G. Coupling an epitaxial quantum dot to a fiber based external-mirror microcavity. Appl. Phys. Lett. 95, 173101 (2009).

7. Greuter, L., Starosielec, S., Kuhlmann, A. V. \& Warburton, R. J. Towards high-cooperativity strong coupling of a quantum dot in a tunable microcavity. Phys. Rev. B 92, 045302 (2015).

8. Fujiwara, M., Toubaru, K., Noda, T., Zhao, H.-Q. \& Takeuchi, S. Highly efficient coupling of photons from nanoemitters into single-mode optical fibers. Nano Lett. 11, 4362 (2011).

9. Liebermeister, L. et al. Tapered fiber coupling of single photons emitted by a deterministically positioned single nitrogen vacancy center. Appl. Phys. Lett. 104, 031101 (2014).

10. Ates, S. et al. Improving the performance of bright quantum dot single photon sources using temporal filtering via amplitude modulation. Sci. Rep. 3, 1397 (2013).

11. Lee, C.-M. et al. Efficient single photon source based on $\mu$-fibre-coupled tunable microcavity. Sci. Rep. 5, 14309 (2015).

12. Patel, R. N. et al. Efficient photon coupling from a diamond nitrogen vacancy center by integration with silica fiber. Light Sci. Appl. 5, e16032 (2016).

13. Daveau, R. S. et al. Efficient fiber-coupled single-photon source based on quantum dots in a photonic-crystal waveguide. Optica 4(2), 178-184 (2017).

14. Burek, M. J. et al. Fiber-coupled diamond quantum nanophotonic interface. Phys. Rev. Appl. 8, 024026 (2017).

15. Xu, X. et al. "Plug and play" single-photon sources. Appl. Phys. Lett. 90, 061103 (2007).

16. Xu, X. et al. "Plug and play" single photons at $1.3 \mu \mathrm{m}$ approaching gigahertz operation. Appl. Phys. Lett. 93, 021124 (2014).

17. Ma, B. et al. Single photon extraction from self-assembled quantum dots via stable fiber array coupling. Appl. Phys. Lett. 110, 142104 (2017).

18. Żołnacz, K. et al. Method for direct coupling of a semiconductor quantum dot to an optical fiber for single-photon source applications. Opt. Express 27, 26772-26785. https://doi.org/10.1364/OE.27.026772 (2019).

19. Schlehahn, A. et al. A stand-alone fiber-coupled single-photon source. Sci. Rep. 8, 1340 (2018)

20. Bremer, L. et al. Quantum dot single-photon emission coupled into single-mode fibers with $3 \mathrm{~d}$ printed micro-objectives. APL Photon. 5, $106101(2020)$

21. Haupt, F. et al. Fiber-connectorized micropillar cavities. Appl. Phys. Lett. 97, 131113 (2010).

22. Snijders, H. et al. Fiber-coupled cavity-qed source of identical single photons. Phys. Rev. Appl. 9, 031002 (2018).

23. Schroder, T., Schel, A. W., Kewes, G., Aichele, T. \& Benson, O. Fiber-integrated diamond-based single photon source. Nano Lett. 11, 198 (2011)

24. Vogl, T., Lu, Y. \& Lam, P. K. Room temperature single photon source using fiber-integrated hexagonal boron nitride. J. Phys. D 50, 295101 (2017)

25. Sasakura, H. et al. Fiber-based bidirectional solid-state single-photon emitter based on semiconductor quantum dot. Appl. Phys. Express 6, 065203 (2013).

26. Zha, G.-W. et al. In situ probing and integration of single self-assembled quantum dots-in-nanowires for quantum photonics. Nanotechnologyhttps://doi.org/10.1088/0957-4484/26/38/385706 (2015).

27. Cadeddu, D. et al. A fiber-coupled quantum-dot on a photonic tip. Appl. Phys. Lett. 108, 011112 (2016).

28. Lee, C.-M. et al. A fiber-integrated nanobeam single photon source emitting at telecom wavelengths. Appl. Phys. Lett. 114, 171101. https://doi.org/10.1063/1.5089907 (2019).

29. Dalacu, D., Poole, P. J. \& Williams, R. L. Nanowire-based sources of non-classical light. Nanotechnology 30, 232001 (2019).

30. Reimer, M. E. et al. Overcoming power broadening of the quantum dot emission in a pure wurtzite nanowire. Phys. Rev. B 93, 195316 (2016).

31. Claudon, J. et al. A highly efficient single-photon source based on a quantum dot in a photonic nanowire. Nat. Photon. 4, 174 (2010).

32. Dalacu, D., Williams, P. J. \& Williams, R. L. Tailoring the geometry of bottom-up nanowires: Application to high efficiency single photon sources. Nanomaterials 11, 1201 (2021).

33. Dalacu, D. et al. Pump power control of photon statistics in a nanowire quantum dot. Phys. Rev. B 102, 115401. https://doi.org/ 10.1103/PhysRevB.102.115401 (2020).

34. Reimer, M. E. et al. Bright single-photon sources in bottom-up tailored nanowires. Nat. Commun. 3, 737 (2012).

35. Zeeshan, M., Sherlekar, N., Ahmadi, A., Williams, R. L. \& Reimer, M. E. Proposed scheme to generate bright entangled photon pairs by application of a quadrupole field to a single quantum dot. Phys. Rev. Lett. 122, 227401 (2019).

36. Laferrière, P. et al. Multiplexed single-photon source based on multiple quantum dots embedded within a single nanowire. Nano Lett. 20, 3688-3693 (2020)

37. Haffouz, S. et al. Bright single InAsP quantum dots at telecom wavelengths in position-controlled InP nanowires: The role of the photonic waveguide. Nano Lett. 18, 3047 (2018).

38. Fiset-Cyr, A. et al. In-situ tuning of individual position-controlled nanowire quantum dots via laser-induced intermixing. Appl. Phys. Lett. 113, 053105 (2018).

39. Laferrière, P. et al. Unity yield of deterministically positioned quantum dot single photon sources. http://arxiv.org/abs/quant-ph/ 2110.08366 (2021)

40. Dalacu, D. et al. Selective-area vapour-liquid-solid growth of InP nanowires. Nanotechnology 20, 395602 (2009).

41. Dalacu, D. et al. Selective-area vapor-liquid-sold growth of tunable InAsP quantum dots in nanowires. Appl. Phys. Lett. 98, 251101 (2011).

\section{Acknowledgements}

This work was supported by the Canadian Space Agency through a collaborative project entitled 'Development of Quantum Dot Based QKD-relevant Light Sources'. 


\section{Author contributions}

D.N. carried out the experiments with D.D.; D.N. analyzed the data. D.D. and R.W. conceived the experiment. J.L. performed the electron beam lithography to define the nanowire patterns. P.P. grew the nanowires and quantum dots. R.W. and G.A. provided theoretical modelling of the optics and correlation statistics. J.P. coded the scanning routine. D.N., D.D. and R.W. devised the device assembly procedures. J.W. assembled the GRIN lenses to fibres. All authored reviewed the manuscript.

\section{Competing interests}

The authors declare no competing interests.

\section{Additional information}

Correspondence and requests for materials should be addressed to D.B.N.

Reprints and permissions information is available at www.nature.com/reprints.

Publisher's note Springer Nature remains neutral with regard to jurisdictional claims in published maps and institutional affiliations.

Open Access This article is licensed under a Creative Commons Attribution 4.0 International

License, which permits use, sharing, adaptation, distribution and reproduction in any medium or format, as long as you give appropriate credit to the original author(s) and the source, provide a link to the Creative Commons licence, and indicate if changes were made. The images or other third party material in this article are included in the article's Creative Commons licence, unless indicated otherwise in a credit line to the material. If material is not included in the article's Creative Commons licence and your intended use is not permitted by statutory regulation or exceeds the permitted use, you will need to obtain permission directly from the copyright holder. To view a copy of this licence, visit http://creativecommons.org/licenses/by/4.0/.

(c) Crown 2021 